Received: 23 January 2017

Accepted: 11 April 2017

Published online: 24 May 2017
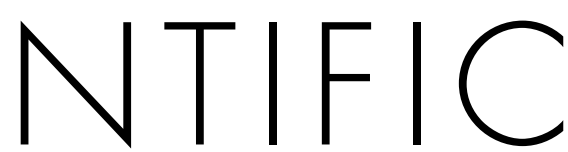

REP

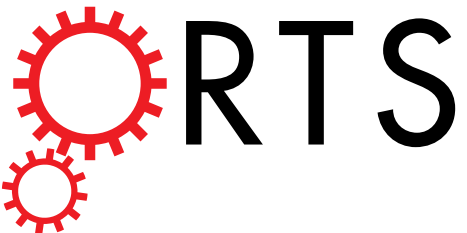

A soluble starch synthase I gene, IbSSI, alters the content, composition, granule size and structure of starch in transgenic

\title{
sweet potato
}

\author{
Yannan Wang, Yan Li, Huan Zhang, Hong Zhai, Qingchang Liu \& Shaozhen He
}

Soluble starch synthase I (SSI) is a key enzyme in the biosynthesis of plant amylopectin. In this study, the gene named IbSSI, was cloned from sweet potato, an important starch crop. A high expression level of $I b S S /$ was detected in the leaves and storage roots of the sweet potato. Its overexpression significantly increased the content and granule size of starch and the proportion of amylopectin by upregulating starch biosynthetic genes in the transgenic plants compared with wild-type plants (WT) and RNA interference plants. The frequency of chains with degree of polymerization (DP) 5-8 decreased in the amylopectin fraction of starch, whereas the proportion of chains with DP 9-25 increased in the IbSSI-overexpressing plants compared with WT plants. Further analysis demonstrated that IbSSI was responsible for the synthesis of chains with DP ranging from 9 to 17 , which represents a different chain length spectrum in vivo from its counterparts in rice and wheat. These findings suggest that the IbSSI gene plays important roles in determining the content, composition, granule size and structure of starch in sweet potato. This gene may be utilized to improve the content and quality of starch in sweet potato and other plants.

In plants, starch consists of amylose and amylopectin. Amylose mainly comprises linear chains that are linked by $\alpha-1,4 \mathrm{O}$-glycosidic bonds, whereas amylopectin is highly branched and contains 5-6\% $\alpha-1,6 \mathrm{O}$-glycosidic bonds to generate glucan branches of various lengths ${ }^{1}$. The biosynthesis of starch in plants is mainly mediated by four classes of enzymes: ADP-glucose pyrophosphorylase (AGPase), starch synthase, starch branching enzyme (SBE) and starch debranching enzyme (DBE) $)^{2}$.

To date, great progress has been achieved in understanding the physicochemical properties of different types of starch synthases and their functions in glucan chain elongation and starch granule formation. Starch synthase can be grouped into five types, granule-bound starch synthase (GBSS) and four types of soluble starch synthases (SS): SSI, SSII, SSIII and SSIV. In cereals, GBSSI is encoded by the Waxy $(w x)$ locus and functions mainly to synthesize amylose ${ }^{3}$, but it also contributes to the synthesis of long amylopectin chains ${ }^{4}$. The loss of GBSSI function does not significantly alter the starch content of rice $^{5}$, maize $^{6}$ or wheat ${ }^{7,8}$, but these mutants show reduced amylose content or a complete lack of amylose. In contrast, SSI, SSII and SSIII are mainly involved in amylopectin synthesis by mediating chain elongation. In rice, three genes encode SSII (SSII-1, 2, 3), two genes encode SSIII $(S S I I I-1,2)$, SSIV (SSIV-1,2) and GBSS (GBSSI, II), and only one gene encodes SSI ${ }^{9}$. The loss of SSII decreases the proportion of amylopectin chains with DP 11-30 and increases the accumulation of amylose ${ }^{10-14}$. The mutation of rice SSIIIa reduces the content of long chains $(\mathrm{DP} \geq 30)$ in the endosperm and results in smaller and rounder starch granules and floury-like endosperm, without affecting the seed starch content ${ }^{15,16}$. Deficiency in maize SSIII decreased the proportion of DP 36 to 53 chains $^{17}$. These results indicate that the elongation of long glucan chains is mainly due to SSIII and not SSII. The number of starch granules per plastid is decreased and the granule

Key Laboratory of Sweet potato Biology and Biotechnology, Ministry of Agriculture/Beijing Key Laboratory of Crop Genetic Improvement/Laboratory of Crop Heterosis and Utilization, Ministry of Education, China Agricultural University, Beijing, 100193, China. Yannan Wang and Yan Li contributed equally to this work. Correspondence and requests for materials should be addressed to Q.L. (email: sunnynba@cau.edu.cn) or S.H. (email: liuqc@cau.edu.cn) 
size is increased in Arabidopsis mutants that carry defective SSIV, which suggests that this gene is involved in the initiation stages of starch granule biosynthesis rather than in the elongation of amylopectin chains ${ }^{18}$. Unlike the other three types of soluble starch synthases (SSII, SSIII and SSIV), which have been found to contain multiple isoforms, SSI has no known isoform ${ }^{19}$. It is suggested that SSI may play a specific role in starch biosynthesis.

In rice, evidence has shown that both in vivo and in vitro SSI mainly synthesizes DP 8-12 chains by adding glucose residues to the short B1 and B2 chains that emerge from the branching points or to the A chains with DP 6-7. Meanwhile, a deficiency in rice SSI does not significantly impact the amylose content or morphology of rice endosperm starch granules, which suggests that other types of SS may compensate for the partial function of SSI ${ }^{2}$. Two mutants generated by T-DNA insertion into the Arabidopsis SSI gene demonstrated that AtSSI was most active on glycogen with an average outer chain length (OCL) of 7-8 compared with maize amylopectin $(\mathrm{OCL}=12-14)$ and maize $\beta$-limit dextrin $(\mathrm{OCL}=2.5)$. This length is consistent with the preferable DP value of 6-7 that is often modified by rice SSI. Meanwhile, the mutant accumulates less starch than WT Arabidopsis ${ }^{1}$. Similarly to AtSSI, maize SSI is most active for glycogen substrates with an OCL ranging from 7 to $9^{20}$.

Sweet potato, Ipomoea batatas (L.) Lam., is an important root crop with a high yield per hectare. It is a mainstay of food consumption in several undeveloped regions because it is a source of high dietary energy for humans and can resist abiotic and biotic stresses ${ }^{21}$. In addition to its contribution to human subsistence, the starchy root of this crop, which can be processed into bio-ethanol, is widely used in the energy industry ${ }^{22}$. Nevertheless, improving the content and quality of sweet potato starch remains an urgent demand, especially in the field of biotechnology.

In sweet potato, several genes that encode the starch biosynthetic enzymes have been cloned and characterized $^{13,14,23-27}$. These genes have been reported to impact the starch composition, structure and physicochemical properties and minimally influence the starch content. However, the role of SSI in starch biosynthesis of sweet potato has not been reported. In this study, we isolated the soluble starch synthase I gene (IbSSI) from the sweet potato. The sweet potato was genetically manipulated to overexpress or suppress $I b S S I$, which affected the content, composition, structure and physicochemical properties of starch. These findings will contribute to the improvement of starch content and quality in sweet potato for use in the biofuel industry.

\section{Results}

Isolation and sequence analysis of IbSSI. The IbSSI gene was cloned from a high-starch sweet potato line Xu 781 using RACE methods. The cloned 2440-bp full-length IbSSI cDNA contained a 1974-bp ORF that produced a polypeptide with a molecular weight of $72.9 \mathrm{kDa}$ and a predicted $\mathrm{pI}$ of 5.34 . A search for the amino acid sequence of IbSSI in NCBI returned a putative glycogen synthase domain that featured an ADP-binding pocket (Supplementary Fig. S1). A multiple alignment of IbSSI with the SSI from potato, Arabidopsis, maize and wheat using DNAMAN revealed three domains that were highly conserved in the plant starch synthases and $E$. coli glycogen synthases ${ }^{28,29}$ (Supplementary Fig. S2). Among the three domains, domain I contained a K- $x$-G-G-L motif which is included in the ADP-glucose binding motif ${ }^{30}$. The binding of ADP-glucose to IbSSI is a key step in elongating the glucan polymers. A phylogenetic analysis further revealed (Fig. 1A) that IbSSI is more closely related to the potato (Solanum tuberosum) StSSI, and the proteins that clustered together with IbSSI all belonged to the soluble starch synthase I family. The genomic sequence of IbSSI was $4027 \mathrm{bp}$ in length. An analysis of the genomic structure using the Spidey program revealed that IbSSI contained 15 exons and 14 introns, and this structure is highly similar to that of potato StSSI. Furthermore, domains I, II and III in IbSSI and StSSI were distributed in the same exons, with domain I in the second exon, domain II in the twelfth exon and domain III spanning the twelfth and thirteenth exons (Fig. 1B). However, the genomic structures of IbSSI and StSSI significantly differed. Each exon of IbSSI is longer than the corresponding StSSI exon, but the total length of the introns in StSSI (6480 bp) is three times the length of introns in IbSSI (2053 bp). These findings suggest that IbSSI may contain less regulatory sequences than StSSI, which may be responsible for their differences in expression patterns and biological functions. The $5^{\prime}$-promoter region $(\sim 1800 \mathrm{bp})$ of IbSSI was isolated and a number of cis-acting regulatory elements were detected by PlantCARE (Supplementary Table S4).

IbSSI activity in E. coli. The cloned IbSSI gene was expressed in E. coli Transetta (DE3) to examine its starch synthase activity. An SDS-PAGE of the cell lysate that contained the IbSSI recombinant protein indicated that IbSSI could be successfully expressed in the prokaryotic system (Supplementary Fig. S3). The specific activity of soluble starch synthase from E. coli cells that contained the recombinant IbSSI ( 29.50 units/mg protein) increased 3.86 -fold compared with those that contained the native pET-28a vector (6.07 units/mg protein) (Supplementary Table S3). This result confirmed that IbSSI encodes a functional starch synthase.

Expression analysis of IbSSI in sweet potato. The spatial expression pattern of IbSSI was investigated by qRT-PCR. The results revealed that IbSSI was expressed in all five major tissues of Xu 781, with much higher levels detected in the leaves and storage roots than in petioles, stems and fibrous roots (Fig. 2A). This expression pattern is similar to that of another starch biosynthetic gene in sweet potato, $I b S B E I I^{31}$.

To examine whether exogenous sucrose treatment could induce the expression of IbSSI, leaf-petioles of Xu 781 were treated with either water (control) or $175 \mathrm{mM}$ sucrose for $48 \mathrm{~h}$. Treatment with water did not up-regulate IbSSI expression in leaf-petioles (Fig. 2B). In contrast, exogenous sucrose treatment could significantly enhance the expression of IbSSI. After $48 \mathrm{~h}$ of treatment, the transcript level of IbSSI showed 9-fold up-regulation in response to the sucrose treatment.

The circadian clock of plants is endogenous and sustained in a cycling manner with a free-running period of approximately $24 \mathrm{~h}^{32}$. A time-course experiment was designed to determine whether expression of IbSSI follows the circadian rhythm (Fig. 2C). The level of IbSSI mRNA was high at $10 \mathrm{am}$ (P2, P5) in the first two normal $24 \mathrm{~h}$ photoperiods and showed severe reduction during the $32 \mathrm{~h}$ extended dark period (P7-P10). This result indicated that the light supply was the external clue that sustained the rhythmic oscillation of IbSSI mRNA level in the 

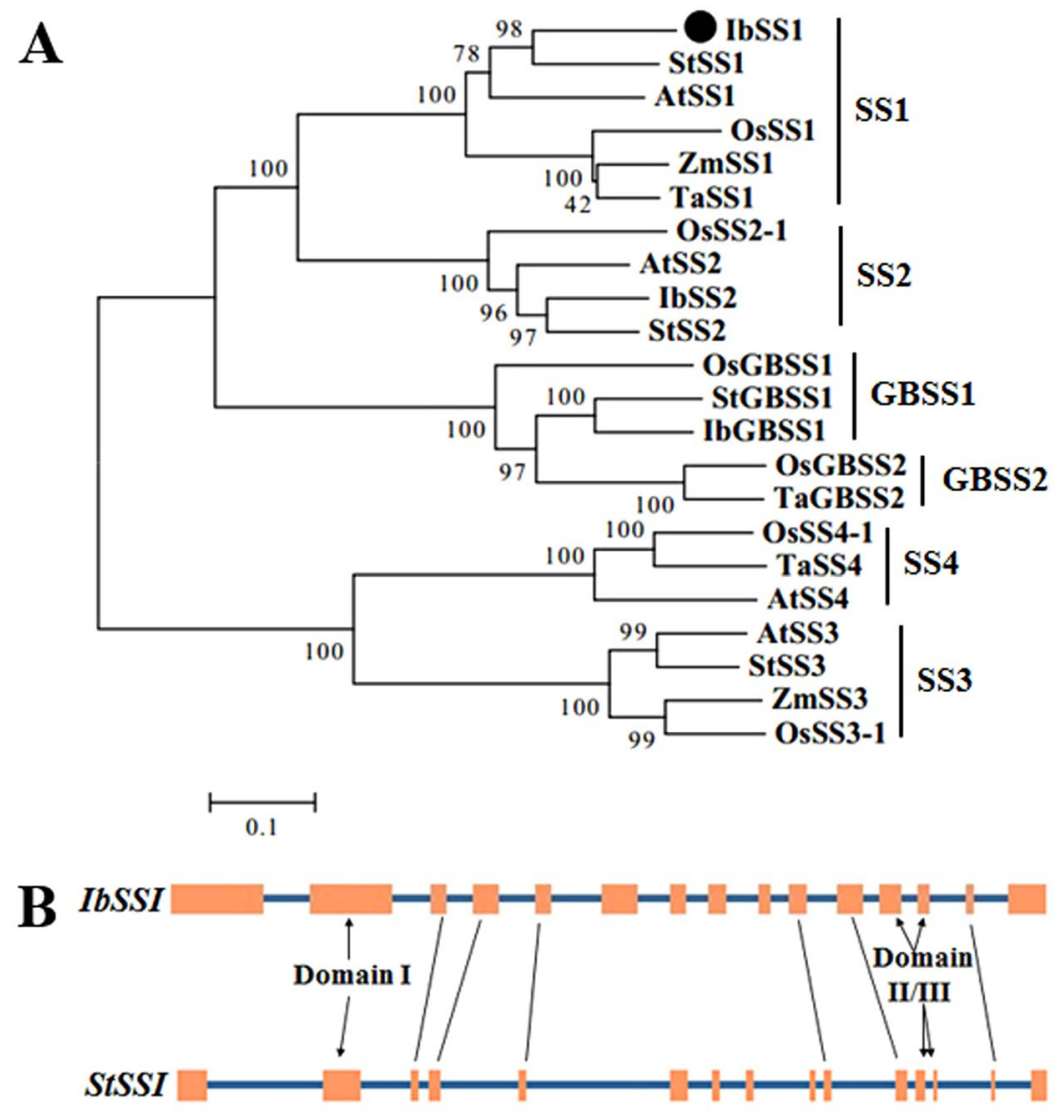

Figure 1. Sequence analysis of IbSSI. (A) Phylogenetic tree of the starch synthases of different plant species generated using MEGA 6.0 with the neighbor-joining method. The IbSSI cloned in this study is indicated by a black spot. The complete species names of the starch synthases and their GenBank accession numbers are listed in Supplementary Table S2. (B) Comparison of the genomic structures of IbSSI (Ipomoea batatas) and StSSI (Solanum tuberosum). The orange boxes represent the exons and the blue lines indicate the introns. Distributions of the three conserved domains are illustrated by black arrows.

normal $24 \mathrm{~h}$ photoperiod and the circadian clock does not regulate the expression of IbSSI. The level of IbSSI mRNA began to restore when the external light was resumed (P11, P12).

Subcellular localization of IbSSI protein. The subcellular localization of IbSSI protein was examined in N. benthamiana epidermal cells and Arabidopsis protoplasts. As shown in Fig. 3, in N. benthamiana epidermal cells, IbSSI was observed as scattered patches and co-localized with the autofluorescence of chloroplasts. In Arabidopsis protoplast, signals associated with the IbSSI were also localized to the chloroplasts. Furthermore, signals of IbSSI were observed to spread throughout the stroma of chloroplasts both in N. benthamiana epidermal cells and Arabidopsis protoplasts, which is similar to the localization pattern of AtSSI in N. benthamiana chloroplasts $^{33}$. These results indicated that IbSSI is localized to chloroplasts, wherein starch biosynthesis occurs.

Production of transgenic sweet potato plants. To functionally characterize $I b S S I$, we constructed its overexpression vector (pC3301-121-IbSSI) and RNAi vector (pFGC5941-IbSSI) (Supplementary Fig. S4) and introduced them into the low-starch sweet potato cultivar Lizixiang, respectively. A total of 217 putative transgenic plants that overexpressed IbSSI were generated (denoted L1, L2, .., L217), and a GUS staining assay and PCR analysis confirmed that IbSSI was overexpressed in 34 of these plants. Furthermore, 125 putative RNAi plants (denoted Li1, Li2, ..., Li125) were obtained, and PCR analysis indicated that IbSSI was successfully knocked down in only 12 of these plants. These 12 RNAi plants (RNAi lines) and another 12 IbSSI-overexpressing plants (overexpression lines) that were randomly selected from the 34 aforementioned plants were planted in the soil in the greenhouse and then in the field. Storage roots were harvested after 5 months of growth in the field. Production of transgenic sweet potato plants is illustrated in Supplementary Fig. S5.

The transcript level of IbSSI in the storage roots of the 24 transgenic lines and WT was investigated by qRT-PCR (Fig. 4). Compared with WT, the transcript level of IbSSI was highest in three overexpression lines (L34, L37 and L132) and lowest in two RNAi lines (Li7 and Li118). These five lines were selected for further analyses.

Amounts of starch-granule-bound proteins. Starch-granule-bound proteins were extracted from $30 \mathrm{mg}$ of starch granules and separated by SDS-PAGE and detected by silver staining (Fig. 5). The major granule-bound proteins detected include GBSSI, SSI, ISA1, SSII, SBEI and SBEII. In WT starch, slight quantity of SSI was detected. 

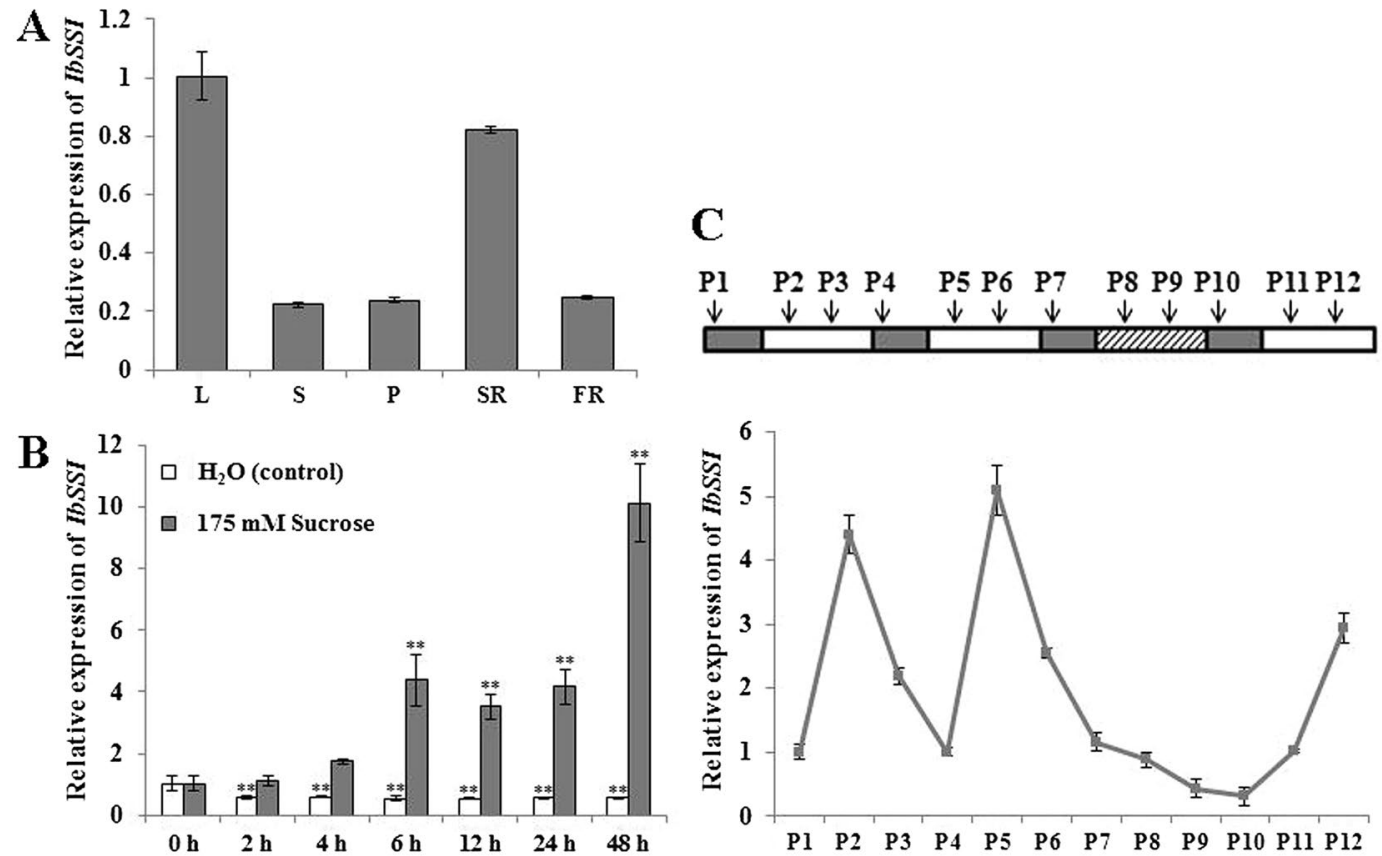

Figure 2. Expression analysis of IbSSI in the sweet potato. (A) Transcript levels of IbSSI in different tissues of the sweet potato detected by qRT-PCR. L, leaf; S, stem; P, petiole; SR, storage root; FR, fibrous root. (B) Expression profile of $I b S S I$ in response to $175 \mathrm{mM}$ sucrose treatment. (C) Time course of IbSSI expression detected by qRT-PCR during $16 \mathrm{~h}$ light/ $8 \mathrm{~h}$ dark photoperiods with light on at $5 \mathrm{am}$ and off at $9 \mathrm{pm}$. Total RNA was extracted from the in vitro-grown whole plant sampled at $10 \mathrm{pm}(\mathrm{P} 1, \mathrm{P} 4, \mathrm{P} 7$ and P10), 10 am (P2, P5, P8 and $\mathrm{P} 11)$ and $4 \mathrm{pm}$ (P3, P6, P9 and P12), respectively. The open bars represent the $16 \mathrm{~h}$ light period, the solid bars correspond to the $8 \mathrm{~h}$ dark period, and the hatched bars indicate the extended dark period that would otherwise be the light period. The results are expressed as relative values with respect to the $\mathrm{L}, 0 \mathrm{~h}$ and $\mathrm{P} 1$, which were set to 1.0 in $(\mathbf{A}-\mathbf{C})$, respectively. Data are presented as the mean $\pm \operatorname{SD}(\mathrm{n}=3) . * *$ Indicates a significant difference versus the control at $P<0.01$ based on Student's $t$-test.

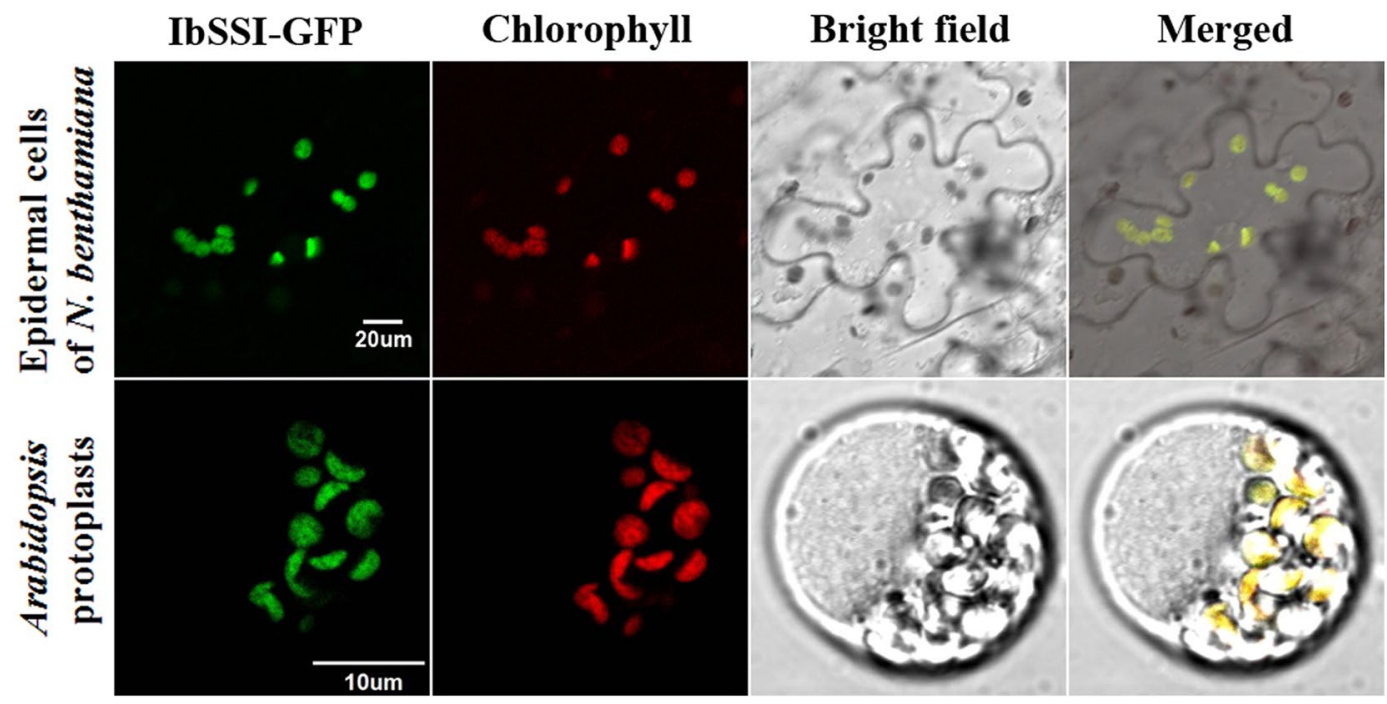

Figure 3. Subcellular localization of IbSSI protein. Upper row: confocal images of green fluorescence of IbSSI-GFP fusion protein are shown in N. benthamiana leaf hypodermal cells, which co-localized with the autofluorescence of chloroplasts. Scale bar $=20 \mu \mathrm{m}$. Lower row: co-localization of IbSSI-GFP signals with autofluorescence of chloroplasts in Arabidopsis mesophyll protoplast. Scale bar $=10 \mu \mathrm{m}$.

The amount of SSI protein increased in the overexpression lines, whereas the two RNAi lines showed no detectable abundance of SSI protein. The other granule-bound proteins remained unchanged in the transgenic lines. 


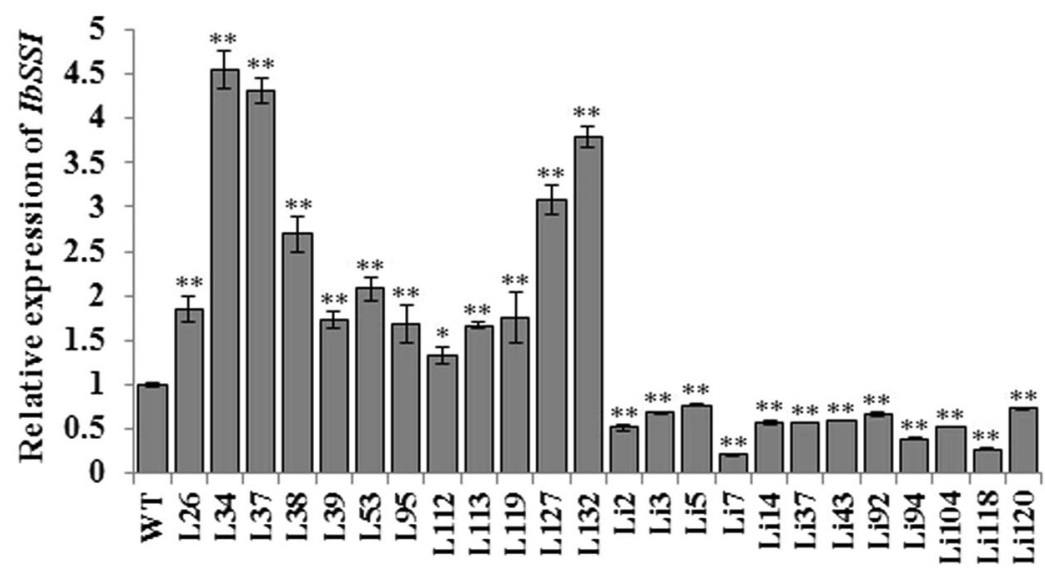

Figure 4. Transcript levels of IbSSI in the storage roots of the 24 transgenic lines and WT. The results are expressed as relative values with respect to WT, which was set to 1.0. Data are presented as the mean $\pm \mathrm{SD}$ $(\mathrm{n}=3)$. *And **Indicate a significant difference versus the $\mathrm{WT}$ at $P<0.05$ and $<0.01$, respectively, based on Student's $t$-test.

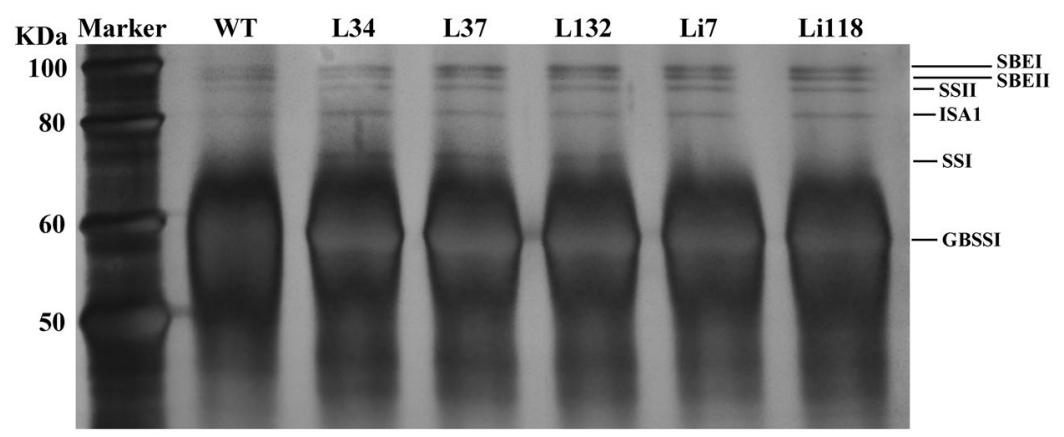

Figure 5. Detection of starch-granule-bound proteins in the transgenic lines and WT. A total of $5 \mathrm{mg}$ of purified starch from each line were boiled in $80 \mu \mathrm{L}$ SDS buffer for $5 \mathrm{~min}$ and centrifuged at 15,000 g. Equal amount of supernatant $(25 \mu \mathrm{L})$ were loaded into the gel. The proteins were visualized by silver staining.

\begin{tabular}{|l|l|l|l|}
\hline \multirow{2}{*}{ Lines } & Starch content & \multicolumn{2}{|l|}{ Relative proportion of amylose (\%) } \\
\cline { 2 - 4 } & $\left(\mathbf{m g ~ g}^{-1} \mathbf{F W}\right)$ & Colorimetric method & Con A method \\
\hline WT & $120.5 \mathrm{~b}(3.0)$ & $19.29 \mathrm{~b}(0.23)$ & $18.45 \mathrm{c}(0.44)$ \\
\hline L34 & $122.1 \mathrm{~b}(3.5)$ & $17.44 \mathrm{c}(0.18)$ & $16.67 \mathrm{e}(0.22)$ \\
\hline L37 & $148.9 \mathrm{a}(1.7)$ & $17.19 \mathrm{c}(0.38)$ & $15.60 \mathrm{f}(0.43)$ \\
\hline L132 & $153.3 \mathrm{a}(2.7)$ & $18.95 \mathrm{~b}(0.14)$ & $17.29 \mathrm{~d}(0.28)$ \\
\hline Li7 & $103.4 \mathrm{c}(3.0)$ & $21.29 \mathrm{a}(0.23)$ & $20.37 \mathrm{~b}(0.30)$ \\
\hline Li118 & $108.3 \mathrm{c}(2.6)$ & $21.68 \mathrm{a}(0.14)$ & $21.03 \mathrm{a}(0.14)$ \\
\hline
\end{tabular}

Table 1. Starch content and composition of the transgenic plants and WT ${ }^{\mathrm{a}}$. ${ }^{\mathrm{D}}$ ata are presented as the mean of three replicates (starch samples from three different storage roots) with standard deviation given in the parenthesis. The values in the same column with different letters differ significantly at $P<0.05$, based on Student's $t$-test.

Starch content and composition. The starch and amylose contents were quantified in the storage roots of the five transgenic lines and WT (Table 1). The starch content was significantly increased in two of the overexpression lines (L37 and L132) and significantly reduced in lines Li7 and Li118 compared with WT. To characterize the starch composition, we used the colorimetric method and Con A method to measure the amylose content. The overexpression of IbSSI significantly reduced the the relative proportion of amylose compared with WT when examined by both methods (Table 1) and the colorimetric measurement was about $4.6-10.2 \%$ higher than the Con A method. In contrast, the relative proportion of amylose was increased in the RNAi lines. These results demonstrate that IbSSI can impact the content and composition of starch in sweet potato. 

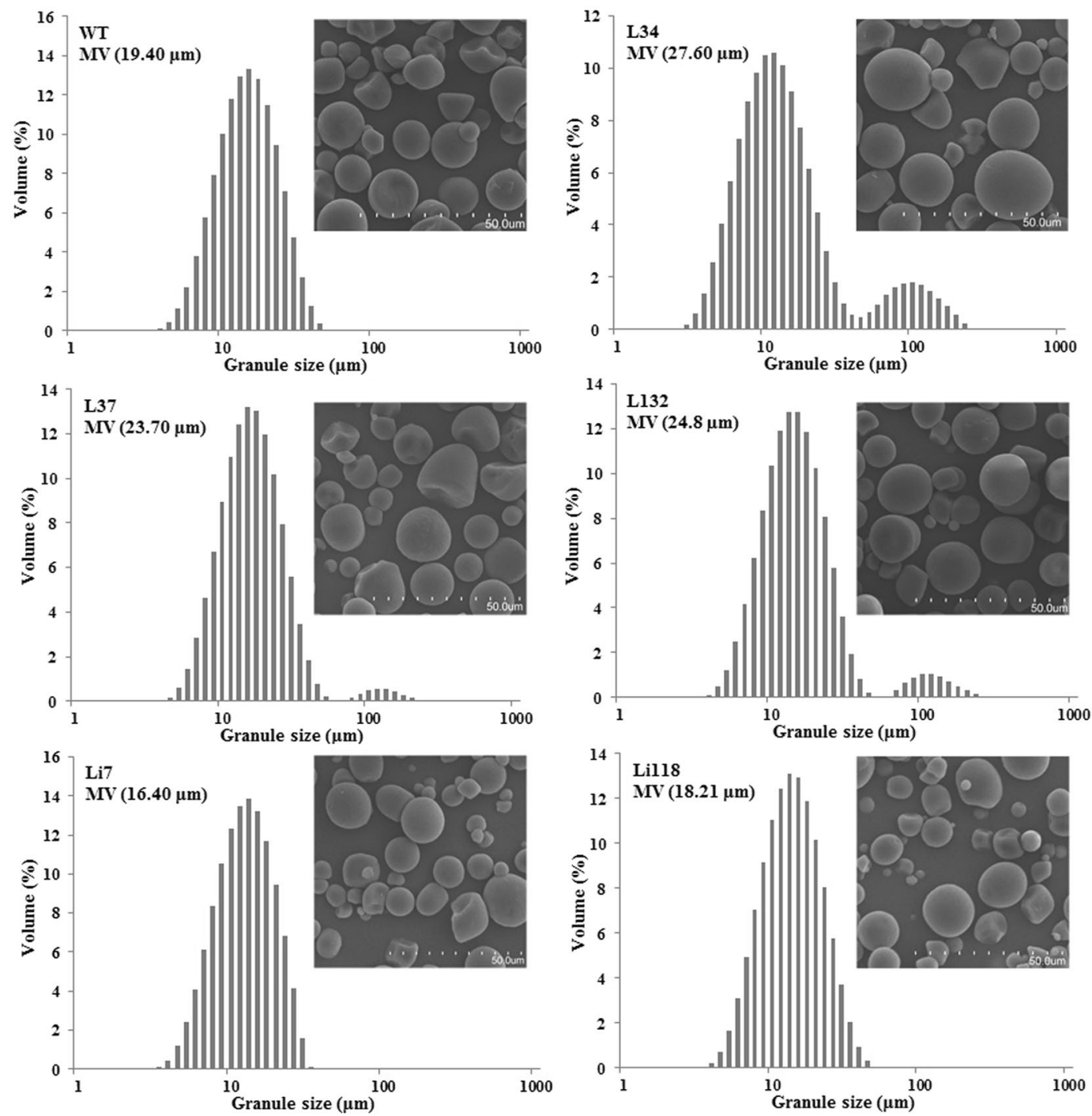

Figure 6. Size distribution and mean volume diameter (MV) of starch granules from the transgenic plants and WT. Insets: Scanning electron micrographs of the starch granules from the transgenic plants and WT. The dotted line indicates a length of $50.0 \mu \mathrm{m}$.

Starch granule size and morphology. As shown in Fig. 6, the starch granule size distributions in the transgenic lines differed from that of WT. Unlike the unimodal patterns observed in the RNAi lines and WT, two peaks appeared in the overexpression lines at approximately $13 \mu \mathrm{m}$ and $104 \mu \mathrm{m}$. The overexpression lines exhibited a broader granule size distribution than WT and contained a greater number of larger starch granules. This part of larger starch granules was largely responsible for the increased average granule size (MV value) in the overexpression lines. In contrast, the average granule size of the RNAi lines was smaller than that of WT because the RNAi lines exhibited a narrower granule size distribution (Li7, size distribution: $2.93-37.66 \mu \mathrm{m}$ ) or a smaller proportion of larger granules (Li118) than WT (size distribution: 3.33-55.24 $\mu \mathrm{m}$ ).

The starch granules that had been purified from the transgenic plants and WT were examined by SEM (Fig. 6, insets). The granules from the overexpression lines were slightly larger than those from WT, whereas the RNAi lines contained a greater number of smaller granules. No other significant morphological alterations were observed in the five lines. Each line contained multiple granular shapes, but the characteristic oval shape was maintained, and no fissures were observed in the granules. These morphological characteristics were similar to those observed by Noda et al. ${ }^{34}$ in sweet potato. Some incomplete-spherical and polygonal granules were detected in each sample, which were likely due to immature development or physical damage during the granule purification process.

Chain length distribution (CLD). The amylopectin CLD of DP 5-70 in the transgenic plants and WT were calculated based on the peak areas (Fig. 7A). The CLD pattern of each line was similar to that of WT. A trough at DP 8 and two peaks at around DP 14 and DP 47 were observed. This pattern is consistent with previous findings ${ }^{22}$. The value obtained for the amylopectin glucan chain of WT was subtracted from the corresponding value for 


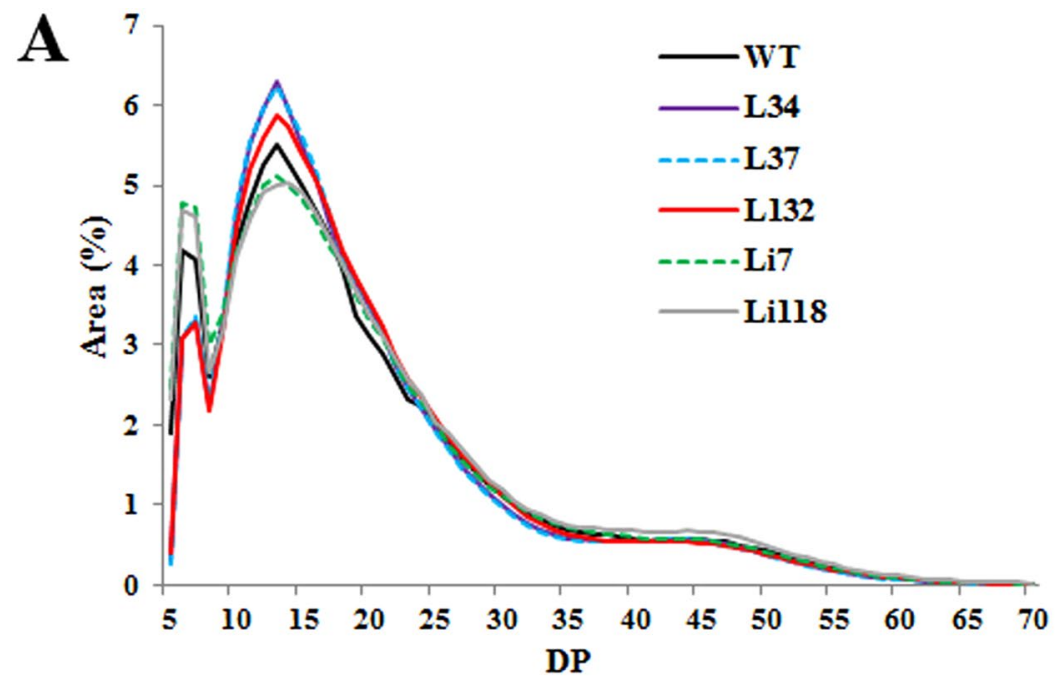

B

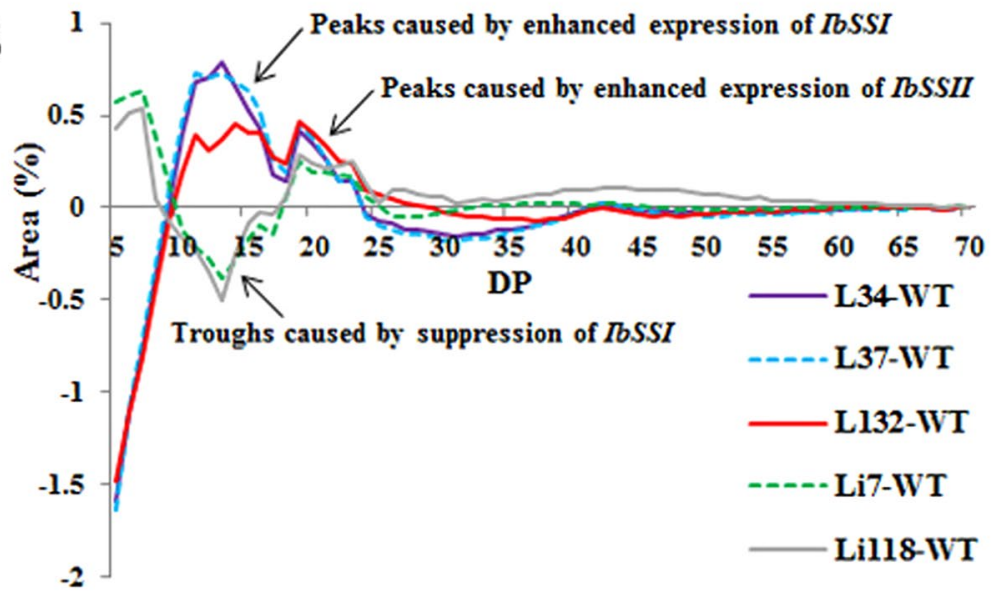

Figure 7. Amylopectin chain length distributions of the transgenic plants and WT. (A) CLD of the starch isolated from the five transgenic lines and WT after normalization to the total peak area. (B) Differences in the CLD between the transgenic lines and WT were calculated as follow: the normalized CLD value for each transgenic line minus the value obtained for the WT.

the transgenic lines to generate the CLD difference model (Fig. 7B). In the overexpression lines, the number of chains with DP 5-8 and DP 26-40 decreased, while the number of chains with DP 9-25 increased. Between DP 9-25, the magnitude of the change peaked at approximately DP 14 and then decreased to a trough until another peak appeared at DP 19. The change in the CLD observed for Li7 and Li118 was almost a mirror image of those observed for L34, L37 and L132 between DP 5-18, which displayed an increase for chains with DP 5-8 and $\mathrm{DP} \geq 18$ and a decrease for chains with DP 9-17. These CLD patterns were different from those in sweet potatoes, in which IbSSII was inhibited ${ }^{13}$, 14 . The opposing CLD alteration patterns between the overexpression and RNAi lines indicated that the IbSSI gene might play a key role in the synthesis of amylopectin chains with DP 9-17 (short to intermediate) in sweet potato.

X-ray diffraction measurement. The X-ray diffractograms of the starch samples from different transgenic lines and WT are presented in Fig. 8 and the degree of crystallinity are shown in parentheses. All the starch samples exhibited A-type crystal patterns with strong reflections at $2 \theta$ of about $15^{\circ}$ and $23^{\circ}$. An unresolved double peak at $2 \theta$ of $17^{\circ}$ and $18^{\circ}$ were also found. This result was similar with the observation in rice, in which the mutation of SSI did not change the A-type crystal pattern of the control line ${ }^{2}$. In WT starch, approximately $43 \%$ crystallinity was detected whereas L34 and L132 showed higher crystallinity. However, reduction of crystallinity was observed in Li7 and Li118. The changes of crystallinity of starch from the transgenic lines were probably caused by the variations in their relative proportion of amylose as revealed by Cheetham and Tao ${ }^{35}$. Even so, the changes in amylose content or starch CLD were not big enough to alter the crystal patterns of the starch from the transgenic lines.

Thermal analysis of starch. The DSC parameters of the starch samples were determined in order to evaluate the gelatinization properties (Table 2). The starch of WT gelatinized with a temperature range of $61.9^{\circ} \mathrm{C}\left(T_{\mathrm{o}}\right)$ to $81.5^{\circ} \mathrm{C}\left(T_{\mathrm{c}}\right)$ and an enthalpy $(\Delta H)$ of $12.1 \mathrm{Jg}^{-1}$. The gelatinization temperatures $\left(T_{\mathrm{o}}, T_{\mathrm{p}}\right.$ and $\left.T_{\mathrm{c}}\right)$ were decreased in the five transgenic lines and showed clear differences in comparison with WT. This tendency is different from 


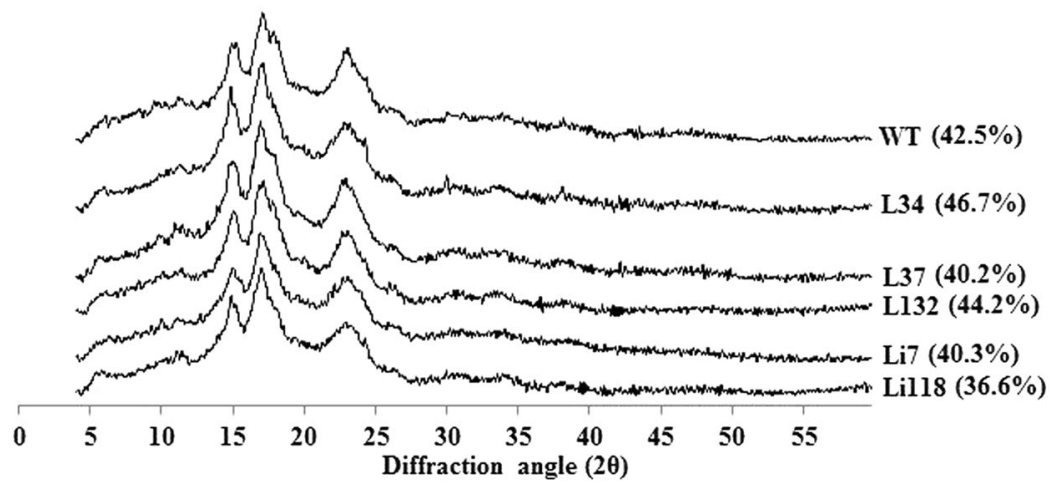

Figure 8. Wide-angle X-ray powder diffraction spectra for starches from WT and different transgenic lines. All the starch samples showed typical A-type crystal pattern. The percentage in the parentheses are the degree of crystallinity.

\begin{tabular}{|l|l|l|l|l|}
\hline Lines & $\boldsymbol{T}_{\mathbf{o}}\left({ }^{\circ} \mathbf{C}\right)$ & $\boldsymbol{T}_{\mathbf{p}}\left({ }^{\circ} \mathbf{C}\right)$ & $\boldsymbol{T}_{\mathbf{c}}\left({ }^{\circ} \mathbf{C}\right)$ & $\Delta \boldsymbol{H}\left(\mathbf{J ~ g}^{-1}\right)$ \\
\hline WT & $61.9 \mathrm{a}(0.1)$ & $72.1 \mathrm{a}(0.1)$ & $81.5 \mathrm{a}(0.5)$ & $12.1 \mathrm{~b}(0.8)$ \\
\hline L34 & $58.8 \mathrm{~b}(0.1)$ & $63.6 \mathrm{~d}(0.2)$ & $81.7 \mathrm{a}(0.5)$ & $15.4 \mathrm{a}(0.7)$ \\
\hline L37 & $58.6 \mathrm{~b}(0.2)$ & $68.8 \mathrm{c}(0.1)$ & $79.6 \mathrm{~b}(0.6)$ & $12.3 \mathrm{~b}(0.6)$ \\
\hline L132 & $57.7 \mathrm{c}(0.2)$ & $70.2 \mathrm{~b}(0.7)$ & $80.8 \mathrm{a}(0.1)$ & $12.1 \mathrm{~b}(1.3)$ \\
\hline Li7 & $57.7 \mathrm{c}(0.1)$ & $61.3 \mathrm{f}(0.3)$ & $69.7 \mathrm{~d}(1.0)$ & $9.0 \mathrm{c}(0.8)$ \\
\hline Li118 & $58.0 \mathrm{c}(0.1)$ & $62.9 \mathrm{e}(0.2)$ & $73.5 \mathrm{c}(0.6)$ & $11.1 \mathrm{~b}(0.5)$ \\
\hline
\end{tabular}

Table 2. DSC analysis of starch from the transgenic plants and WTa ${ }^{a}$ Data are presented as the mean of three replicates with standard deviation given in the parenthesis. The values in the same column with different letters differ significantly at $P<0.05$, based on Student's $t$-test.

\begin{tabular}{|l|l|l|l|l|l|l|l|}
\hline Lines & Peak viscosity $(\mathbf{c P})$ & $\begin{array}{l}\text { Hot paste } \\
\text { viscosity }(\mathbf{c P})\end{array}$ & Breakdown $(\mathbf{c P})$ & Final viscosity $(\mathbf{c P})$ & Setback $(\mathbf{c P})$ & Peak time $(\mathbf{m i n})$ & $\begin{array}{l}\text { Pasting } \\
\text { temperature }\left({ }^{(} \mathbf{C}\right)\end{array}$ \\
\hline WT & $3331.7 \mathrm{c}(7.6)$ & $1895.0 \mathrm{~b}(18.0)$ & $1463.3 \mathrm{c}(22.5)$ & $2619.7 \mathrm{a}(29.1)$ & $724.7 \mathrm{bc}(19.7)$ & $10.2 \mathrm{c}(0.0)$ & $70.7 \mathrm{a}(0.4)$ \\
\hline L34 & $3207.0 \mathrm{~d}(12.2)$ & $1684.3 \mathrm{c}(29.8)$ & $1522.7 \mathrm{~b}(26.0)$ & $2418.0 \mathrm{~b}(22.5)$ & $733.7 \mathrm{~b}(8.0)$ & $10.1 \mathrm{c}(0.0)$ & $70.0 \mathrm{a}(0.1)$ \\
\hline L37 & $2809.7 \mathrm{e}(23.7)$ & $1315.7 \mathrm{~d}(23.8)$ & $1494.0 \mathrm{~b}(47.5)$ & $1962.7 \mathrm{c}(25.5)$ & $647.0 \mathrm{~d}(18.4)$ & $9.9 \mathrm{~d}(0.2)$ & $67.8 \mathrm{~b}(0.6)$ \\
\hline L132 & $2685.3 \mathrm{f}(36.9)$ & $1293.3 \mathrm{~d}(17.9)$ & $1392.0 \mathrm{c}(19.0)$ & $1874.7 \mathrm{~d}(24.5)$ & $581.3 \mathrm{e}(7.37)$ & $9.8 \mathrm{~d}(0.0)$ & $66.9 \mathrm{c}(0.4)$ \\
\hline Li7 & $3489.7 \mathrm{~b}(11.5)$ & $1961.0 \mathrm{a}(3.5)$ & $1528.7 \mathrm{~b}(12.1)$ & $2650.3 \mathrm{a}(27.0)$ & $689.3 \mathrm{c}(23.7)$ & $10.5 \mathrm{~b}(0.0)$ & $66.1 \mathrm{~d}(0.5)$ \\
\hline Li118 & $3928.3 \mathrm{a}(29.7)$ & $1855.3 \mathrm{~b}(32.9)$ & $2073.0 \mathrm{a}(20.9)$ & $2625.0 \mathrm{a}(23.1)$ & $769.7 \mathrm{a}(31.1)$ & $10.7 \mathrm{a}(0.0)$ & $65.1 \mathrm{e}(0.5)$ \\
\hline
\end{tabular}

Table 3. RVA analysis of starch from the transgenic plants and WTa ${ }^{\mathrm{a}}$ Data are presented as the mean of three replicates with standard deviation given in the parenthesis. The values in the same column with different letters differ significantly at $P<0.05$, based on Student's $t$-test.

that observed by Fujita et al. ${ }^{2}$ and McMaugh et al. ${ }^{36}$ in rice and wheat. In their studies, suppression of SSI gene led to $1^{\circ} \mathrm{C}$ to $3{ }^{\circ} \mathrm{C}$ higher gelatinization temperatures. Compared with WT, L34 starch had significantly higher $\Delta H$ while Li7 starch showed lower $\Delta H$ during gelatinization. No significant differences in $\Delta H$ were demonstrated for starch from other transgenic lines.

Pasting properties of starch. The pasting properties of starch were analyzed by the RVA and the results are presented in Table 3. The pasting temperatures of the five transgenic lines were lower than that of WT. The peak viscosity $(\mathrm{PV})$ values were lower in the overexpression lines and higher in the RNAi lines than in WT. The breakdown (BD) value did not differ between the five lines and WT. And the values for hot paste viscosity (HV), final viscosity (FV) and setback (SB) were significantly lower in the overexpression lines than in WT, while no typical pattern was identified for the RNAi lines.

Expression of starch biosynthetic genes and enzyme activity assay. The transcript levels of 11 starch biosynthetic genes in the five transgenic lines were examined by qRT-PCR (Fig. 9). Six of the 11 genes were up-regulated in the overexpression lines and down-regulated in the RNAi lines (IbAGP-sTL1, IbAGP-sTL2, $I b A G P L I, I b S B E I, I b S B E I I, I b I s a 1)$. In contrast, IbSSIII and IbSSIV showed lower transcript levels in the overexpression lines and higher transcript levels in the RNAi lines, while the transcript levels of IbGBSSI and IbSSII were increased in all the five transgenic lines. The IbPUL gene was significantly up-regulated in two of the 

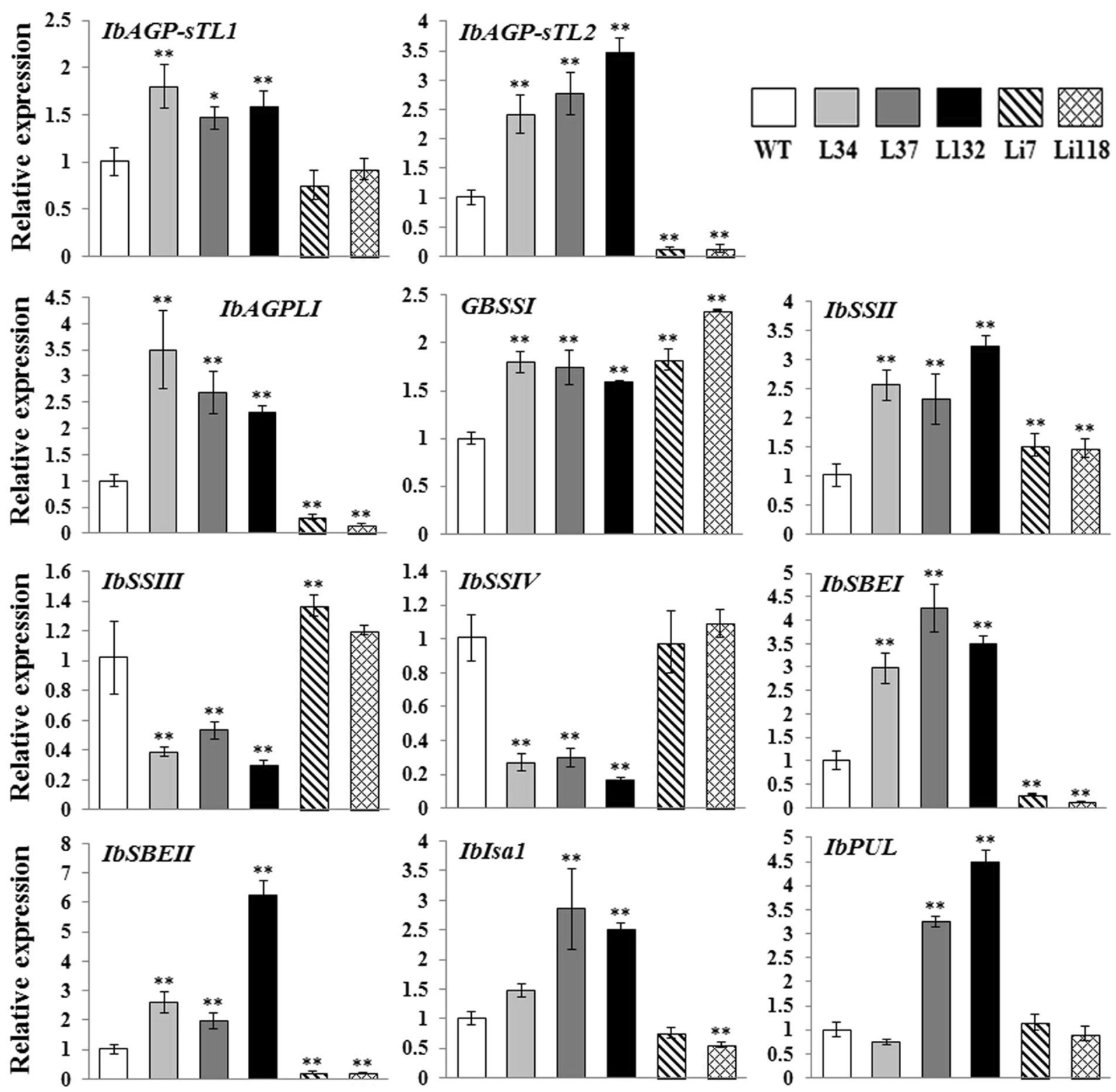

Figure 9. Transcript levels of 11 starch biosynthetic genes in the storage roots of the transgenic plants and WT. The results are expressed as relative values with respect to $\mathrm{WT}$, which was set to 1.0. Data are presented as the means $\pm \mathrm{SD}(\mathrm{n}=3) . *$ And $* *$ Indicate a significant difference versus $\mathrm{WT}$ at $P<0.05$ and $<0.01$, respectively, based on Student's $t$-test.

overexpression lines (L37, L132) and showed no significant difference in transcript levels in lines L34, Li7 and Li118 compared with WT.

The enzyme activity levels of the four starch biosynthetic enzymes (SS, GBSS, AGPase and SBE) depended on the transgenic lines (Fig. 10). The activity of SS was significantly higher in the overexpression lines and lower in the RNAi lines compared with WT. The activity of another starch synthase, GBSS, was enhanced in all transgenic lines. The activities of both SBE and AGPase were increased in the overexpression lines and reduced in the RNAi lines compared with WT. The changes in the activity of each enzyme in transgenic plants were basically consistent with the alterations in the transcript levels of their corresponding genes.

\section{Discussion}

In sweet potato, the spatial expression pattern of IbSSI showed similarity to that of IbSBEII and major difference from that of other starch biosynthetic genes from sweet potato, such as IbAGP-sTL1, IbAGP-sTL2, IbGBSSI and $I b S B E I^{23,24,31,37}$. In potato, the mRNA level of StSSI is drastically lower in tubers than that in sink and source leaves, which might suggest a minor role of StSSI in starch biosynthesis in the storage organs ${ }^{38}$. However, the present study indicated that the transcript level of IbSSI was much higher in leaves and storage roots than in 


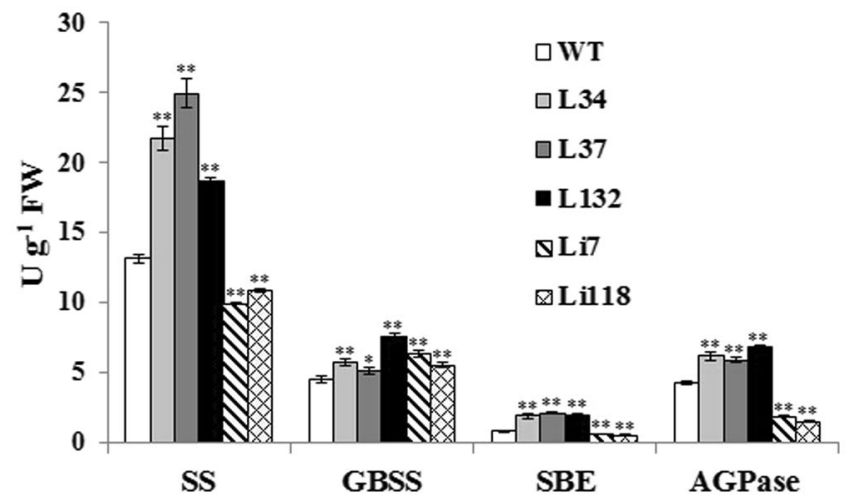

Figure 10. Enzyme activities of SS, GBSS, AGPase, SBE in the storage roots of the transgenic plants and WT. Data are presented as the mean $\pm \mathrm{SD}(\mathrm{n}=3)$. *And **Indicate a significant difference versus $\mathrm{WT}$ at $P<0.05$ and $<0.01$, respectively, based on Student's $t$-test.

petioles, stems and fibrous roots (Fig. 2A). This suggests that IbSSI plays active roles in the starch biosynthesis in both leaves and storage roots.

Sucrose is an important regulatory signal involved in starch metabolism ${ }^{39}$. Starch synthesis is induced when the supply of carbohydrates (either endogenous or exogenous) exceeds the respiratory demand ${ }^{40}$. In the present study, pre-treatment with water in the dark depleted the endogenous carbohydrate and reduced the respiration to a low level. Consequently, floating the leaf-petiole in sucrose triggered starch synthesis and induced IbSSI gene expression. A similar trend was observed for the sweet potato genes IbSpo, IbGBSSI and IbAGP-sTL123, 41,42 . Furthermore, a sucrose-responsive cis-acting regulatory element (Carbohydrate Metabolite Signal Responsive Element 1, CMSRE 1, TGGACGG) was detected at the -379 position of the $5^{\prime}$-promoter region of IbSSI (Supplementary Table S4). This element has been shown to be the core motif that determines the response of several other genes to sucrose ${ }^{43-45}$ and may also play a role in the response of IbSSI to sucrose treatment. Both experimental and sequence analyses collectively indicated that $I b S S I$ is sucrose inducible.

Starch and amylose content are important agronomic traits for root crops. Many studies have been conducted to investigate the regulatory effects of SS genes on these two traits ${ }^{10,15,16,18,38,46}$. The SS genes in these studies were down regulated by RNAi or mutated by T-DNA insertion, which showed no clear rules of alterations in starch and amylose contents. This lack of rule was attributed to the following. Firstly, SS are responsible for the synthesis of amylopectin and the down-regulation of either type of $S S$ may affect the amylose/amylopectin ratio without modifying the total starch content, provided that the total carbon influx does not change. Secondly, other type of SS could functionally compensate for the down-regulation or mutation of one specific gene (for example, overlapping functions of SSII and SSIII in Arabidopsis ${ }^{12}$ ). Thirdly, environmental factors may play a role. In the present study, the overexpression and suppression of IbSSI oppositely affected the starch and amylose content. As shown in Figs 9 and 10, rather than being suppressed as a result of the overexpression of IbSSI, the expression level of IbGBSSI and its enzymatic activity were increased in lines L34, L37 and L132, which likely facilitated the synthesis of amylose. This phenomenon probably occurred because of siphon-like regulation: the overexpression of IbSSI increased the consumption of carbon resources for the synthesis of amylopectin, and this over-exhaustion may have increased the carbon flux into the starch biosynthetic pathway, which up-regulated the expression of $I b A G P$ and IbGBSSI. However, the increase in expression of IbGBSSI was smaller than the overall increase in expression of $I b S S I$ and $I b S S I I$ (Figs 9 and 10), and the unbalanced increase in IbGBSSI and IbSSI (and IbSSII) expression decreased the relative proportion of amylose. Additionally, the up-regulation of the transcript level and enzymatic activity of $I b S B E$ in the overexpression lines also decreased the relative proportion of amylose, as described by Kitahara et al. ${ }^{27}$. Moreover, the collective enhancement of the activities of AGPase, GBSS and SS contributed to the accumulation of starch in the storage roots of the overexpression lines. The RNAi lines exhibited different activity patterns in the starch biosynthetic genes. The activity of AGPase was significantly decreased in RNAi lines, which reduced the starch content. The activity of SS in Li7 and Li118 showed significantly but relatively small reduction compared WT, likely due to the enhanced activity of SSIII and/or SSIV. The increase in the GBSS activity and the decrease in the SBE activity may together result in the accumulation of amylose in these two lines.

In the present study, the suppression of IbSSI increased the proportion of chains with DP 5-8 and DP $\geq 18$ and reduced the proportion of chains with DP 9-17. Thus, we assume that the DP 5-8 chains accumulated because they were not elongated efficiently by IbSSI to DP 9-17 chains, whose proportion decreased subsequently. Meanwhile, the available DP 9-17 chains were further converted to longer chains with DP 18-25 by IbSSII, which was up-regulated in the RNAi lines (Fig. 9). This was similar with the rice SSI mutant, in which the deficiency of OsSSI leaves more short chains with DP 8-12 suitable for OsSSII to generate longer chains Fujita et al. ${ }^{2}$. Previous study showed that maize SSI displayed an affinity for an average chain length of DP 7-14 and its affinity for chains of DP $>20$ is significantly lower because it becomes entrapped within the starch granule $e^{20}$. In this study, overexpression of IbSSI increased the amount of chains with DP 9-25 and decreased the chains with DP 5-8, with two peaks and a trough appearing at DP 13, DP 19 and DP 17, respectively (Fig. 7B). Accordingly, it is suggested that in sweet potato, DP 18 represents the time at which IbSSI became inactive. Based on this assumption, chains 
with DP 5-8 in the overexpression lines decreased because the elevated levels of IbSSI converted these chains to additional DP 9-17 chains. Subsequently, these chains were further elongated by IbSSII to DP 18-25 chains. Thus, the up-regulation of IbSSI and IbSSII in the overexpression lines gave rise to the two peaks at DP 13 and DP 19. Collectively, these results suggest that IbSSI is mainly responsible for the synthesis of amylopectin chains with DP 9-17, which is different from the preferable DP spectrum of SSI from rice $(8-12)$ and wheat $(8-12)^{2,36}$. Concurrently, the transgenic lines contained some slight alterations in chains with DP 26-55 compared with WT, which could be attributed to the alteration in expression levels of IbSSIII and/or IbSSIV (Fig. 9), as described in previous studies ${ }^{16,47,48}$. Nevertheless, additional evidence is needed to explain the changes in CLD in the transgenic lines based on the mechanism underlying the interaction between the starch biosynthetic genes.

The altered expression of IbSSI in the transgenic lines changed the size distribution of the starch granules, possibly due to the significantly differed amylose content. In the Arabidopsis SSI and rice SSIIIa mutant lines ${ }^{1,27}$, enrichment of amylose content caused more smaller starch granules, which was similar to lines Li7 and Li118 in the present study. Conversely, the average granule size positively correlated with the amylose content in the overexpression lines (L34, L37 and L132). It is noteworthy that a new fraction of larger granules appeared in the overexpression lines, which may imply an alteration in the starch granule initiation caused by enzymatic changes of SSIII or SSIV as revealed by Szydlowski et al. ${ }^{49}$. And this maybe was another reason that the average size of the starch granules was increased. The Morphological analysis of the starch granules in the five lines by SEM revealed no significant differences compared with the WT. The morphological features of starch granules in rice SSI mutant lines deficient in OSSSI did not significantly differ from those WT rice ${ }^{2}$. In contrast, the starch granules from the potato SSIII mutant lines were deeply fissured on the hilum ${ }^{47}$. Therefore, the IbSSI gene could impact granule size by influencing the synthesis of amylopectin or amylose. A deficiency in the IbSSI did not cause defects in granule morphology, likely because of the overlapping functions provided by other types of soluble starch synthase.

Different types of starch crystalline structures have been found in root and tuber plants. The starch from cassava exhibits the A-type crystallinity ${ }^{50}$, whereas potato and yam are the B-type ${ }^{51,52}$. Both A-type and C-type were reported in different cultivars of sweet potato $22,53-55$. In the present study, the crystal structure of starches in transgenic lines and WT was A-type. The present results further indicated that the peak viscosity values were higher in the RNAi lines and lower in the overexpression lines than in WT. This result is inconsistent with those of previous studies. The PV of the rice SSI mutant line was $77 \%$ of that of the control line ${ }^{2}$. Many studies of crops that produce waxy starches, such as waxy wheat and waxy barley ${ }^{56,57}$, have demonstrated that a reduction of the amylose content increased the PV value of starch. However, the PV value of waxy potato was lower than that of normal potato $\operatorname{starch}^{58}$, and this was similar to the decreased PV values observed in the overexpression lines in this study. Meanwhile, the lower PV values in the overexpression lines may also be due to their starch containing more intermediate chains (DP 13-25) and less short chains (DP 5-8), which was similar to the lower PV value of the wheat SSI suppression line ${ }^{36}$. No typical patterns were identified for HV, FV and SB values in the RNAi lines, which was likely due to the pleiotropic regulation of IbSSI on other starch metabolic genes, or some other factors were involved such as lipid content of the starch.

In conclusion, the IbSSI gene has been successfully isolated from a high starch sweet potato line Xu 781. Its overexpression significantly increased the starch content and granule size, and decreased the amylose/amylopectin ratio in transgenic sweet potato plants. This gene is also responsible for the synthesis of short to intermediate chains with DP 9-17. These results suggest that IbSSI has great potential to be used to improve the content and quality of starch in sweet potatoes and other plants.

\section{Methods}

Plant materials. The sweet potato line Xu 781 with high starch content was employed for the isolation of the IbSSI gene. A low-starch sweet potato cultivar Lizixiang was used to characterize the function of this gene through determining if its starch could be improved or not. Both $\mathrm{Xu} 781$ and Lizixiang were cultivated in a greenhouse under a regimen of $16 \mathrm{~h} \mathrm{light} / 8 \mathrm{~h}$ dark at $28^{\circ} \mathrm{C}$.

Isolation of IbSSI cDNA, genomic DNA sequences and 5'-promoter region. Total RNA was extracted from the leaves of Xu 781 and transcribed into first-strand cDNA with the PrimeScript II $1^{\text {st }}$ Strand cDNA Synthesis Kit (TaKaRa, Dalian, China). A pair of degenerate primers (DS-F/R) was designed based on the most homologous region identified by aligning the SSI sequence from different plant species. An EST fragment was amplified from the first-strand cDNA. Subsequently, rapid amplification of cDNA ends (RACE) was conducted to obtain the full-length cDNA with gene-specific primers (GSPs). The genomic sequence of IbSSI was amplified with GS-F/R primers using genomic DNA extracted from the leaves of Xu 781 as a template. The $5^{\prime}$-promoter region of IbSSI gene was isolated using the Universal GenomeWalker 2.0 Kit (TaKaRa, Dalian, China). GWS1 and GWS2 were used as gene specific primers. The sequences of the primers used in this study are listed in Supplementary Table S1.

Sequence analysis of IbSSI. The open-reading frame (ORF) of the cloned IbSSI gene was predicted using the ORF Finder (http://www.ncbi.nlm.nih.gov/projects/gorf/). The homology of the IbSSI protein was identified using the protein blast in NCBI (http://blast.ncbi.nlm.nih.gov/Blast.cgi). The molecular weight and theoretical isoelectric point ( $\mathrm{pI}$ ) of the IbSSI protein were calculated using http://web.expasy.org/compute_pi/. A multiple sequence alignment of the IbSSI protein with other SSI proteins was conducted using the DNAMAN software. The genomic structure of IbSSI was analyzed with the Spidey program (http://www.ncbi.nlm.nih.gov/spidey/). A phylogenic tree was constructed using the MEGA 6.0 software with the neighbor-joining (NJ) method. The 
cis-acting regulatory elements in the $5^{\prime}$-promoter region of IbSSI were searched using PlantCARE (http://bioinformatics.psb.ugent.be/webtools/plantcare/html/).

Assessment of IbSSI activity in Escherichia coli. The ORF of IbSSI was amplified using the ES-F/R primers and inserted into the pET-28a vector. Next, the sequence-validated pET-28a-IbSSI vector and native pET-28a vector were introduced into competent E. coli strain Transetta (DE3) cells (Transgen Biotech, Beijing, China). Positive clones were grown in Luria-Bertani (LB) medium. Fresh LB medium was inoculated with the overnight cultures at a 100:1 (v:v) dilution. Soluble cytoplasmic proteins were prepared from isopropyl $\beta$-D-thiogalactopyranoside (IPTG)-induced Transetta (DE3) cells ${ }^{59}$. The expressed IbSSI protein was subjected to SDS-PAGE analysis according to the method of Jiang et al. ${ }^{60}$, and a starch synthase assay was conducted as described previously ${ }^{61}$. One unit of activity was defined as the formation of $1 \mathrm{nmol}$ of ADP per min at $30^{\circ} \mathrm{C}$.

Expression analysis of IbSSI in the sweet potato. The transcript levels of $I b S S I$ were analyzed in five different tissues (storage root, fibrous root, stem, leaf and petiole) of Xu 781 grown for 100 days in a field. Total RNA was extracted from each tissue and reverse transcribed into cDNA and quantitative real-time PCR (qRT-PCR) was conducted using the SYBR detection protocol (TaKaRa) with the ABI 7500 Real-Time PCR system. The primers used to amplify IbSSI and IbActin (endogenous control) are listed in Supplementary Table S1.

The response of IbSSI to exogenous sucrose was investigated as described by Wang et al.$^{42}$ with some modifications. Leaf-petioles $(10 \mathrm{~cm})$ from Xu 781 grown for 100 days in a field were cultured for 1 day in water in the dark and then treated with either water (control) or $175 \mathrm{mM}$ sucrose in the dark at $28^{\circ} \mathrm{C}$. qRT-PCR was conducted to determine the mRNA levels of IbSSI in cuttings harvested at different time points $(0,2,4,6,12,24$ and $48 \mathrm{~h})$ after treatment.

The response of IbSSI to changes of light conditions was examined as described by Leterrier et al. ${ }^{62}$. Twelve in vitro-grown plants of $\mathrm{Xu} 781$ were acclimated to a 16-h-light/8-h-dark regimen in a growth chamber at $28^{\circ} \mathrm{C}$ for 1 month prior to modifying the light conditions. Total RNA was extracted from each whole plant sampled at different time points (shown in Fig. 2C) and the mRNA levels of IbSSI were subsequently detected by qRT-PCR.

Subcellular localization of the IbSSI protein. To construct the IbSSI-GFP fusion protein, the ORF of IbSSI was amplified from the first-strand cDNA with primers $83 \mathrm{~S}-\mathrm{F} / \mathrm{R}$. The sequence-validated ORF fragment was then ligated into the pMDC83 vector. This recombinant vector was introduced into the Agrobacterium tumefaciens strain EHA105 and used for transient expression in Nicotiana benthamiana leaf epidermal cells according to the method of Strasser et al. ${ }^{63}$. Meanwhile, Arabidopsis mesophyll protoplasts were also used for the subcellular localization of IbSSI. Protoplast isolation and vector transfection were conducted as described previously ${ }^{64}$. After co-cultivation, the agroinfiltrated tobacco leaves and transfected Arabidopsis protoplasts were visualized with a laser scanning confocal microscope (Nikon Inc., Melville, NY, USA).

Production of transgenic sweet potato plants. The ORF of IbSSI was amplified using OS-F/R primers and then inserted into pBI121 between the $\mathrm{XbaI}$ and $\mathrm{SacI}$ sites to replace the glucuronidase (gus $A$ ) gene. Next, the expression cassette 35S-IbSSI-NOS was excised from the pBI121-IbSSI vector with HindIII and EcoRI and then ligated between the same cleavage sites in pCAMBIA3301 to generate the overexpression vector. To construct the RNA interference (RNAi) plasmid, two fragments (FDF and RDF) were amplified from the coding sequence of IbSSI using the primers Si-UF/R and Si-DF/R and inserted into the cleavage sites of XhoI/SwaI and BamHI/XbaI in the pFGC5941 vector, respectively. The sequence of the recombinant vector pFGC5941-IbSSI was validated. The two recombinant plasmids for overexpression and RNAi were transfected into A. tumefaciens strain EHA105, respectively. Embryogenic suspension cultures of Lizixiang used for Agrobacterium infection were established with the method of Liu et al. ${ }^{65}$. The procedures for embryogenic transformation, selection of resistant calluses and plant regeneration were as described by Liu et al. ${ }^{66}$ except that phosphinothricin and cefotaxime sodium were added in the selection culture.

The putatively transgenic sweet potato plants overexpressing IbSSI were identified using the histochemical GUS assay according to Jefferson et al. ${ }^{67}$. The blue staining of tissues indicated a positive reaction. Genomic DNA was extracted from the leaves of these GUS-positive plants, and PCR amplifications were performed using the primers T35-F and TS-R to further confirm that these plants were transgenic. To identify the IbSSI RNAi plants, genomic DNA was extracted from the leaves of plantlets regenerated from the calluses, and PCR was conducted using the primers int-F/R.

Expression of starch biosynthetic genes. The transcript levels of $I b S S I$ and 11 starch biosynthetic genes in the storage roots of the transgenic plants and wild-type plants (WT) were investigated by qRT-PCR. The starch biosynthetic genes included the following: IbAGP-sTL1 and 2 (encoding the two small subunits of IbAGPase, respectively), IbAGP-TLI (encoding a large subunit of IbAGPase), IbGBSSI, IbSSII, IbSSIII, IbSSIV, IbSBEI, IbSBEII, IbIsa1 and IbPUL. The primers used to amplify these genes are listed in Supplementary Table S1.

Analysis of starch-granule-bound proteins. Starch-granule-bound proteins were isolated and separated by SDS-PAGE as described previously ${ }^{68}$. After electrophoresis, the proteins were visualized using the Pierce Silver Stain Kit (Thermo Fisher Scientific).

Enzyme activity assay in storage roots. The activity of four starch biosynthetic enzymes (SS, GBSS, AGPase and SBE) in the storage roots of the transgenic plants and WT was evaluated as described by Nakamura et al. ${ }^{69}$. One unit of activity (SS, GBSS and AGPase) was defined as the formation of $1 \mathrm{nmol} \mathrm{ADP}$ per min at $30^{\circ} \mathrm{C}$ and 1 unit of SBE activity was defined as the amount of enzyme required to increase the spectrophotometric absorbance by 1 unit in 1 minute. 
Quantification of starch content and composition. The starch content in the storage roots of WT and transgenic plants was analyzed according to the method of Smith and Zeeman ${ }^{70}$. Starch was isolated from the sweet potato storage roots as described by Zhao et al..$^{50}$. Fresh slices of sweet potato tuberous roots were suspended in distilled water and homogenized in a blender, and the slurry was filtered through a $100-\mu \mathrm{m}$ sieve. The supernatant was discarded after the sedimentation of the starch granules. This process was repeated three times, and the starch samples were dried in a convection oven at $40^{\circ} \mathrm{C}$ for 2 days. The amylose was then quantified using the colorimetric amylose content assay ${ }^{71}$. Standard curves were established with the standard samples of potato amylose and amylopectin (Sigma). Meanwhile, the Concanavalin A (Con A) method was also used to determine the relative proportion of amylose using an amylose/amylopectin assay kit (Megazyme International Ireland).

Analysis of starch granule size and morphology. The granule size distribution of starch from storage root was determined as described by Zhou et al. ${ }^{22}$. A Master-size 2000 laser diffraction instrument (Malvern Instruments Ltd., Worcestershire, UK) was used in wet-well mode. The starch was added to the reservoir and sonicated for $30 \mathrm{~s}$ at $6 \mathrm{~W}$ until an obscuration value of $12-17 \%$ was achieved. The refractive indices used for the water and starch were 1.330 and 1.50, respectively. The particle size distributions are presented as the diameter versus volume $\mathrm{e}^{72}$. To examine whether the starch granule morphology was altered in different transgenic lines, the starch granules were coated with gold after they were spread on a metal stub and then observed under a scanning electron microscope (SEM).

Analysis of the chain length distribution (CLD). The CLD was measured according to Zhou et al..$^{22}$. Starch from the transgenic plants and WT was digested with Pseudomonas amyloderamosa isoamylase (Sigma), and the CLD of amylopectin was analyzed using high-performance anion-exchange chromatography with pulsed amperometric detection (HPAEC-PAD; Dionex-ICS 3000; Dionex Corporation, USA).

Analysis of starch granule crystallinity. A D8 Advance Bruker X-ray diffractometer (Bruker AXS, Germany) was used to study the crystal type of the starch granules. Starch powders were scanned with the $2 \theta$ diffraction angle at $4-50^{\circ}$. The crystal patterns and degree of crystallinity were analyzed using Jade 5.0 software.

Analysis of thermal characteristics. The thermal properties of the starch samples were examined using a differential scanning calorimeter (DSC Q2000; TA Instrument Ltd., UK). A mixture of starch and distilled water $(\mathrm{w} / \mathrm{w}, 1: 3)$ were sealed in an aluminum pan and scanned at $10^{\circ} \mathrm{C} \mathrm{min}{ }^{-1}$ from $30^{\circ} \mathrm{C}$ to $95^{\circ} \mathrm{C}$ with an empty aluminum pan as a reference. The gelatinization temperature and enthalpy were calculated using the Universal Analysis 2000 software.

Measurement of pasting properties. The pasting properties of the starch were analyzed using a rapid viscosity analyzer (model RVA-Super 3; Newport Scientific Pty. Ltd., Australia). The starch was suspended in distilled water $(10 \% \mathrm{w} / \mathrm{v}$, dry weight basis, $25 \mathrm{~mL})$ and tested using the integrated sweet potato program. The temperature of the starch slurry was increased from $30^{\circ} \mathrm{C}$ to $95^{\circ} \mathrm{C}$ at a rate of $5^{\circ} \mathrm{C} \mathrm{min}-1$ and held at $95^{\circ} \mathrm{C}$ for $6 \mathrm{~min}$, followed by cooling to $50^{\circ} \mathrm{C}$ at the same rate and maintenance for $10 \mathrm{~min}$. The rotating speed of the paddle remained constant $(160 \mathrm{rpm})$ throughout the analysis, excluding the speed of $960 \mathrm{rpm}$ applied during the first $10 \mathrm{~s}$.

\section{References}

1. Delvallé, D. et al. Soluble starch synthase I: a major determinant for the synthesis of amylopectin in Arabidopsis thaliana leaves. Plant J. 43, 398-412, doi:10.1111/tpj.2005.43.issue-3 (2005).

2. Fujita, N. et al. Function and characterization of starch synthase I using mutants in rice. Plant Physiol. 140, 1070-1084, doi:10.1104/ pp.105.071845 (2006)

3. James, M. G., Denyer, K. \& Myers, A. M. Starch synthesis in the cereal endosperm. Curr. Opin. Plant Bio. 6, 215-222, doi:10.1016/ S1369-5266(03)00042-6 (2003).

4. Denyer, K., Clarke, B., Hylton, C., Tatge, H. \& Smith, A. M. The elongation of amylose and amylopectin chains in isolated starch granules. Plant J. 10, 1135-1143, doi:10.1046/j.1365-313X.1996.10061135.x (1996).

5. Sano, Y. Differential regulation of waxy gene expression in rice endosperm. Theor. Appl. Genet. 68, 467-473, doi:10.1007/ BF00254822 (1984).

6. Tsai, C. Y. The function of the waxy locus in starch synthesis in maize endosperm. Biochem. Genet. 11, 83-96, doi:10.1007/ BF00485766 (1974).

7. Fujita, N., Hasegawa, H. \& Taira, T. The isolation and characterization of a waxy mutant of diploid wheat (Triticum monococcum L.). Plant Sci. 160, 595-602, doi:10.1016/S0168-9452(00)00408-8 (2001).

8. Nakamura, T., Yamamori, M., Hirano, H., Hidaka, S. \& Nagamine, T. Production of waxy (amylose-free) wheats. Mol. Genet. Genomics 248, 253-259, doi:10.1007/BF02191591 (1995).

9. Hirose, T. \& Terao, T. A comprehensive expression analysis of the starch synthase gene family in rice (Oryza sativa L.). Planta 220, 9-16, doi:10.1007/s00425-004-1314-6 (2004).

10. Craig, J. et al. Mutations in the gene encoding starch synthase II profoundly alter amylopectin structure in pea embryos. Plant Cell 10, 413-426 (1998).

11. Yamamori, M., Fujita, S., Hayakawa, K., Matsuki, J. \& Yasui, T. Genetic elimination of a starch granule protein, SGP-1, of wheat generates an altered starch with apparent high amylose. Theor. Appl. Genet. 101, 21-29, doi:10.1007/s001220051444 (2000).

12. Zhang, X. L. et al. Overlapping functions of the starch synthases SSII and SSIII in amylopectin biosynthesis in Arabidopsis. BMC Plant Biol. 8, 96, doi:10.1186/1471-2229-8-96 (2008).

13. Takahata, Y. et al. Inhibition of the expression of the starch synthase II gene leads to lower pasting temperature in sweetpotato starch. Plant Cell Rep. 29, 535-543, doi:10.1007/s00299-010-0842-8 (2010).

14. Kitahara, K. et al. Starch properties of transgenic sweetpotato plants modified by RNA interference of the starch synthase II gene. J. Appl. Glycosci. 58, 85-90, doi:10.5458/jag.jag.JAG-2010_025(2011).

15. Ryoo, N. et al. Knockout of a starch synthase gene OsSSIIIa/Flo5 causes white-core floury endosperm in rice (Oryza sativa L.). Plant Cell Rep. 26, 1083-1095, doi:10.1007/s00299-007-0309-8 (2007).

16. Fujita, N. et al. Characterization of SSIIIa-deficient mutants of rice: the function of SSIIIa and pleiotropic effects by SSIIIa deficiency in the rice endosperm. Plant Physiol. 144, 2009-2023, doi:10.1104/pp.107.102533 (2007). 
17. Lin, Q. H. et al. Functional interactions between starch synthase III and isoamylase-type starch-debranching enzyme in maize endosperm. Plant Physiol. 158, 679-692, doi:10.1104/pp.111.189704 (2012).

18. Roldán, I. et al. The phenotype of soluble starch synthase IV defective mutants of Arabidopsis thaliana suggests a novel function of elongation enzymes in the control of starch granule formation. Plant J. 49, 492-504, doi:10.1111/tpj.2007.49.issue-3 (2007).

19. Jeon, J. S., Ryoo, N., Hahn, T. R., Walia, H. \& Nakamura, Y. Starch biosynthesis in cereal endosperm. Plant Physiol. Biochem. 48, 383-392, doi:10.1016/j.plaphy.2010.03.006 (2010).

20. Commuri, P. D. \& Keeling, P. L. Chain-length specificities of maize starch synthase I enzyme: studies of glucan affinity and catalytic properties. Plant J. 25, 475-486, doi:10.1046/j.1365-313x.2001.00955.x (2001).

21. Mukhopadhyay, S. K., Chattopadhyay, A., Chakraborty, I. \& Bhattacharya, I. Crops that feed the world 5. Sweetpotato. Sweetpotatoes for income and food security. Food Secur. 3, 283-305, doi:10.1007/s12571-011-0134-3 (2011).

22. Zhou, W. Z. et al. Impact of amylose content on starch physicochemical properties in transgenic sweet potato. Carbohyd. Polym. 122, 417-427, doi:10.1016/j.carbpol.2014.11.003 (2015).

23. Bae, J. M. \& Liu, J. R. Molecular cloning and characterization of two novel isoforms of the small subunit of ADPglucose pyrophosphorylase from sweet potato. Mol. Genet. Genomics 254, 179-185, doi:10.1007/s004380050406 (1997).

24. Wang, S. J., Yeh, K. W. \& Tsai, C. Y. Molecular characterization and expression of starch granule-bound starch synthase in the sink and source tissues of sweet potato. Physiol. Plantarum 106, 253-261, doi:10.1034/j.1399-3054.1999.106301.x (1999).

25. Kimura, T. et al. Absence of amylose in sweet potato [Ipomoea batatas (L.) Lam.] following the introduction of granules-bound starch synthase I cDNA. Plant Cell Rep. 20, 663-666 (2001).

26. Kim, S. H., Hamada, T., Otani, M. \& Shimada, T. Cloning and characterization of sweetpotato isoamylase gene (IbIsa1) isolated from tuberous root. Breeding Sci. 55, 453-458, doi:10.1270/jsbbs.55.453 (2005).

27. Kitahara, K. et al. Physicochemical properties of amylose-free and high-amylose starches from transgenic sweetpotato modified by RNA interference. Carbohyd. Polym. 69, 233-240, doi:10.1016/j.carbpol.2006.09.025 (2007).

28. Van der Leij, F. R., Visser, R. G. F., Ponstein, A. S., Jacobsen, E. \& Feenstra, W. J. Sequence of the structural gene for granule-bound starch synthase of potato (Solanum tuberosum L.) and evidence for a single point deletion in the amf allele. Mol. Genet. Genomics 228, 240-248, doi:10.1007/BF00282472 (1991).

29. Baba, T. et al. Identification, cDNA cloning, and gene expression of soluble starch synthase in rice (Oryza sativa L.) immature seeds. Plant Physiol. 103, 565-573, doi:10.1104/pp.103.2.565 (1993).

30. Furukawa, K., Tagaya, M., Inouye, M., Preiss, J. \& Fukui, T. Identification of lysine 15 at the active site in Escherichia coli glycogen synthase (Conservation of a Lys- $X$-Gly-Gly sequence in the bacterial and mammalian enzymes.). J. Biol. Chem. 265, 2086-2090 (1990).

31. Qin, H., Zhou, S. \& Zhang, Y. Z. Characterization and expression analysis of starch branching enzymes in sweet potato. J. Integr. Agr. 12, 1530-1539, doi:10.1016/S2095-3119(13)60369-X (2013).

32. Hillman, W. S. Biological rhythms and physiological timing. Ann. Rev. Plant Physiol. 27, 159-179, doi:10.1146/annurev. pp.27.060176.001111 (1976).

33. Gámez-Arjona, F. M., Raynaud, S. \& Ragel, P. \& Mérida, Á. Starch synthase 4 is located in the thylakoid membrane and interacts with plastoglobule-associated proteins in Arabidopsis. Plant J. 80, 305-316, doi:10.1111/tpj.12633 (2014).

34. Noda, T., Takahata, Y., Sato, T., Hisamatsu, M. \& Yamada, T. Physicochemical properties of starches extracted from sweet potato roots differing in physiological age. J. Agr. Food Chem. 43, 3016-3020, doi:10.1021/jf00060a005 (1995).

35. Cheetham, N. W. H. \& Tao, L. P. Variation in crystalline type with amylose content in maize starch granules: an X-ray powder diffraction study. Carbohyd. Polym. 36, 277-284, doi:10.1016/S0144-8617(98)00007-1 (1998).

36. McMaugh, S. J. et al. Suppression of starch synthase I expression affects the granule morphology and granule size and fine structure of starch in wheat endosperm. J. Exp. Bot. 65, 2189-2201, doi:10.1093/jxb/eru095 (2014).

37. Hamada, T., Kim, S. H. \& Shimada, T. Starch-branching enzyme I gene (IbSBEI) from sweet potato (Ipomoea batatas); molecular cloning and expression analysis. Biotechnol. Lett. 28, 1255-1261, doi:10.1007/s10529-006-9083-x (2006).

38. Kossmann, J., Abel, G. J. W., Springer, F., Lloyd, J. R. \& Willmitzer, L. Cloning and functional analysis of a cDNA encoding a starch synthase from potato (Solanum tuberosum L.) that is predominantly expressed in leaf tissue. Planta 208, 503-511, doi:10.1007/ s004250050587 (1999).

39. Ahn, Y. O. et al. Exogenous sucrose utilization and starch biosynthesis among sweetpotato cultivars. Carbohyd. Res. 345, 55-60, doi:10.1016/j.carres.2009.08.025 (2010).

40. Kossmann, J., Visser, R. G. F., Müller-Röber, B., Willmitzer, L. \& Sonnewald, U. Cloning and expression analysis of a potato cDNA that encodes branching enzyme: evidence for co-expression of starch biosynthetic genes. Mol. Genet. Genomics 230, 39-44, doi:10.1007/BF00290648 (1991).

41. Hattori, T., Fukumoto, H., Nakagawa, S. \& Nakamura, K. Sucrose-induced expression of genes coding for storage root storage protein, sporamin, of sweet potato in leaves and petioles. Plant Cell Physiol. 32, 79-86 (1991).

42. Wang, S. J., Yeh, K. W. \& Tsai, C. Y. Regulation of starch granule-bound starch synthase I gene expression by circadian clock and sucrose in the source tissue of sweet potato. Plant Sci. 161, 635-644, doi:10.1016/S0168-9452(01)00449-6 (2001).

43. Morikami, A. et al. Two cis-acting regulatory elements are involved in the sucrose-inducible expression of the sporamin gene promoter from sweet potato in transgenic tobacco. Mol. Genet. Genomics 272, 690-699, doi:10.1007/s00438-004-1100-y (2005).

44. Maeo, K. et al. Sugar-responsible elements in the promoter of a gene for $\beta$-amylase of sweet potato. Plant Mol. Biol. 46, 627-637, doi:10.1023/A:1010684908364 (2001).

45. Mita, S., Suzuki-Fujii, K. \& Nakamura, K. Sugar-inducible expression of a gene for $\beta$-amylase in Arabidopsis thaliana. Plant Physiol. 107, 895-904, doi:10.1104/pp.107.3.895 (1995).

46. Morell, M. K. et al. Barley sex6 mutants lack starch synthase IIa activity and contain a starch with novel properties. Plant J. 34, 173-185, doi:10.1046/j.1365-313X.2003.01712.x (2003).

47. Fulton, D. C. et al. Role of granule-bound starch synthase in determination of amylopectin structure and starch granule morphology in potato. J. Biol. Chem. 277, 10834-10841, doi:10.1074/jbc.M111579200 (2002).

48. Ral, J. P. et al. Circadian clock regulation of starch metabolism establishes GBSSI as a major contributor to amylopectin synthesis in Chlamydomonas reinhardtii. Plant Physiol. 142, 305-317, doi:10.1104/pp.106.081885 (2006).

49. Szydlowski, N. et al. Starch granule initiation in Arabidopsis requires the presence of either class IV or class III starch synthase. Plant Cell 21, 2443-2457, doi:10.1105/tpc.109.066522 (2009).

50. Zhao, S. S., Dufour, D., Sánchez, T., Ceballos, H. \& Zhang, P. Development of waxy cassava with different biological and physicochemical characteristics of starches for industrial applications. Biotechnol. Bioeng. 108, 1925-1935, doi:10.1002/bit.23120 (2011).

51. Gernat, C., Radosta, S., Damaschun, G. \& Schierbaum, F. Supramolecular structure of legume starches revealed by X-Ray scattering. Starch-Starke 42, 175-178, doi:10.1002/(ISSN)1521-379X (1990).

52. Moorthy, S. N. Physicochemical and functional properties of tropical tuber starches: a review. Starch-Starke 54, 559-592, doi:10.1002/(ISSN)1521-379X (2002).

53. Noda, T., Takahata, Y., Sato, T., Hisamatsu, M. \& Yamada, T. Physicochemical properties of starches extracted from sweet potato roots differing in physiological age. J. Agric. Food Chem. 43, 3016-3020, doi:10.1021/jf00060a005 (1995).

54. Lee, B. H. \& Lee, Y. T. Physicochemical and structural properties of different colored sweet potato starches. Starch-Starke 68, 1-9 (2016). 
55. Takeda, Y., Tokunaga, N., Takeda, C. \& Hizukuri, S. Physicochemical properties of sweet potato starches. Starch-Starke 38, 345-350, doi:10.1002/(ISSN)1521-379X (1986).

56. Yoo, S. H. \& Jane, J. L. Structural and physical characteristics of waxy and other wheat starches. Carbohyd. Polym. 49, 297-305, doi:10.1016/S0144-8617(01)00338-1 (2002).

57. Song, Y. \& Jane, J. Characterization of barley starches of waxy, normal, and high amylose varieties. Carbohyd. Polym. 41, 365-377, doi:10.1016/S0144-8617(99)00098-3 (2000).

58. McPherson, A. E. \& Jane, J. Comparison of waxy potato with other root and tuber starches. Carbohyd. Polym. 40, 57-70, doi:10.1016/ S0144-8617(99)00039-9 (1999).

59. Yan, H. B. et al. The gene encoding starch synthase IIc exists in maize and wheat. Plant Sci. 176, 51-57, doi:10.1016/j. plantsci.2008.09.003 (2009)

60. Jiang, H. W., Dian, W. M., Liu, F. Y. \& Wu, P. Molecular cloning and expression analysis of three genes encoding starch synthasein II rice. Planta 218, 1062-1070, doi:10.1007/s00425-003-1189-y (2004).

61. Harn, C. et al. Isolation and characterization of the $z$ SSIIa and $z$ SSIIb starch synthase cDNA clones from maize endosperm. Plant Mol. Biol. 37, 639-649, doi:10.1023/A:1006079009072 (1998).

62. Leterrier, M., Holappa, L. D., Broglie, K. E. \& Beckles, D. M. Cloning, characterization and comparative analysis of a starch synthase IV gene in wheat: functional and evolutionary implications. BMC Plant Biol. 8, 98, doi:10.1186/1471-2229-8-98 (2008).

63. Strasser, R. et al. Enzymatic properties and subcellular localization of Arabidopsis $\beta$-N-Acetylhexosaminidases. Plant Physiol. 145, 5-16, doi:10.1104/pp.107.101162 (2007).

64. Yoo, S. D., Cho, Y. H. \& Sheen, J. Arabidopsis mesophyll protoplasts: a versatile cell system for transient gene expression analysis. Nat. Protoc. 2, 1565-1572, doi:10.1038/nprot.2007.199 (2007).

65. Liu, Q. C., Zhai, H., Wang, Y. \& Zhang, D. P. Efficient plant regeneration from embryogenic suspension cultures of sweetpotato. In Vitro Cell. Dev. Biol.-Plant 37, 564-567, doi:10.1007/s11627-001-0098-7 (2001).

66. Liu, D. G. et al. Overexpression of IbP5CR enhances salt tolerance in transgenic sweetpotato. Plant Cell Tiss. Org. Cult. 117, 1-16, doi:10.1007/s11240-013-0415-y (2014).

67. Jefferson, R. A., Kavanagh, T. A. \& Bevan, M. W. GUS fusions: $\beta$-glucuronidase as a sensitive and versatile gene fusion marker in higher plants. EMBO J. 6, 3901-3907 (1987).

68. Nakamura, T., Vrinten, P., Hayakawa, K. \& Ikeda, J. Characterization of a granule-bound starch synthase isoform found in the pericarp of wheat. Plant Physiol. 118, 451-459, doi:10.1104/pp.118.2.451 (1998).

69. Nakamura, Y., Yuki, K., Park, S. Y. \& Ohya, T. Carbohydrate metabolism in the developing endosperm of rice grains. Plant Cell Physiol 30, 833-839, doi:10.1093/oxfordjournals.pcp.a077813 (1989).

70. Smith, A. M. \& Zeeman, S. C. Quantification of starch in plant tissues. Nat. Protoc. 1, 1342-1345, doi:10.1038/nprot.2006.232 (2006).

71. Zhu, F., Yang, X. S., Cai, Y. Z., Bertoft, E. \& Corke, H. Physicochemical properties of sweetpotato starch. Starch-Stärke 63, 249-259, doi:10.1002/star.v63.5 (2011).

72. Blazek, J. \& Copeland, L. Pasting and swelling properties of wheat flour and starch in relation to amylose content. Carbohyd. Polym. 71, 380-387, doi:10.1016/j.carbpol.2007.06.010 (2008).

\section{Acknowledgements}

We thank Prof. Peng Zhang for facilitating the characterization of the starch. We also thank Prof. Xiangfeng Wang for his valuable comments on the preliminary draft of this manuscript. This research was supported by the National Natural Science Foundation of China (Grant no. 31371680), the Beijing Food Crops Innovation Consortium Program and the China Agriculture Research System (CARS-11).

\section{Author Contributions}

Y.W. and Y.L. performed most of the experiments and drafted the manuscript. H.Z. and H.Z. participated in data analysis and revised the manuscript. Q.L. and S.H. conceived and designed the experiments and wrote most of the article. All authors have read and approved the final manuscript.

\section{Additional Information}

Supplementary information accompanies this paper at doi:10.1038/s41598-017-02481-X

Competing Interests: The authors declare that they have no competing interests.

Publisher's note: Springer Nature remains neutral with regard to jurisdictional claims in published maps and institutional affiliations.

(c) (i) Open Access This article is licensed under a Creative Commons Attribution 4.0 International License, which permits use, sharing, adaptation, distribution and reproduction in any medium or format, as long as you give appropriate credit to the original author(s) and the source, provide a link to the Creative Commons license, and indicate if changes were made. The images or other third party material in this article are included in the article's Creative Commons license, unless indicated otherwise in a credit line to the material. If material is not included in the article's Creative Commons license and your intended use is not permitted by statutory regulation or exceeds the permitted use, you will need to obtain permission directly from the copyright holder. To view a copy of this license, visit http://creativecommons.org/licenses/by/4.0/.

(C) The Author(s) 2017 